\title{
Establishment of Ferricyanide Chronoamperometric Total Antioxidant Capacity Assay Employing a Carbon Screen-Printed Disposable Microchip -Fundamental Study Using Vegetable Extraction-
}

\author{
Masao Gotoh ${ }^{1, *}$, Hideo Hirose, Tomoko Ishikawa ${ }^{1}$, \\ Hideaki Nakamura $^{2}$ and Kenji Yokoyama ${ }^{1}$ \\ Graduate School of Bionics, Computer and Media Sciences, Tokyo University of Technology, \\ 1404-1 Katakura, Hachioji, Tokyo 192-0982, Japan \\ ${ }^{1}$ Research Center of Advanced Bionics (RCAB), National Institute of Advanced Industrial Science \\ and Technology (AIST), Tokyo University of Technology, \\ 1404-1 Katakura, Hachioji,Tokyo 192-0982, Japan \\ ${ }^{2}$ Department of Liberal Arts, Tokyo University of Technology, 5-23-22, \\ Nishikamata, Ota-ku, Tokyo 144-8535, Japan
}

(Received February 3, 2015; accepted June 17, 2015)

Key words: microchip, antioxidant, methanol, solubility valance, vegetable

In the present study, a carbon screen-printed disposable microchip as a microstructured two-electrode system was developed for the establishment of a ferricyanide chronoamperometric total antioxidant capacity (FC-TAC) assay for vegetables. Ferricyanide cyclic voltammetry (FCV) measurements were also performed using the microchip to define optimum conditions for the FC-TAC assay. As antioxidants, we chose ascorbic acid (vitamin $\mathrm{C}$ ), chlorogenic acid, and $\alpha$-tocopherol (vitamin E). The electrochemical response to an antioxidant was indirectly determined through ferricyanide and was strongly affected by the solubility of both the mediator and the antioxidant in the measurement solution. By intensive investigation, it was found that $80 \%(\mathrm{v} / \mathrm{v})$ methanol was the best solvent for the $\mathrm{FC}$ measurements and vegetable extraction. Under optimum conditions for the FC-TAC assay, micro-volume measurement of $30 \mu \mathrm{L}$ without reaction time was realized using the microchip, and calibration curves for three antioxidants were successfully obtained with excellent determination coefficients $\left(r^{2}>0.986, n=5\right)$. Finally, the conventional DPPH test using ten vegetable extractions was applied for comparison with the present FC-TAC method, and the results obtained showed good correspondence $\left(r^{2}>0.928, n=5\right)$.

${ }^{*}$ Corresponding author: e-mail: gotoh@stf.teu.ac.jp 


\section{Introduction}

Microstructures of biosensors and chemical sensors have been required to reduce costs and sample volume and to enhance disposability. The disposability of a microstructured chip has been studied especially in the fields of diagnoses and food analyses. In our group, several approaches have been tested for this aim. ${ }^{(1)}$ For example, lactic acid in serum was determined with a $0.2 \mu \mathrm{L}$ sample volume and a compactly integrated flow microchip, which was fabricated by micromachining. ${ }^{(2)}$ Further enhancement of the disposability was realized by carbon screen printing onto the surface of a polymer sheet. Using this technique, several types of disposable microchips for electrochemical chronoamperometric sensing employing ferricyanide mediation have been developed for measurements of blood constituents, ${ }^{(3-6)}$ ethanol in alcoholic beverages, ${ }^{(7)}$ environmental water quality, ${ }^{(8)}$ and for composting. ${ }^{(9)}$ From these experiences, we have subsequently developed an disposable electrochemical microchip fabricated by carbon screen printing for ferricyanide chronoamperometry (FC).

In histological biosensor development, the first generation of an electrochemical glucose biosensor has been attempted using a dissolved oxygen electrode..$^{(1,10)}$ Then oxygen was employed as a natural electrochemical mediator. However, the low solubility of oxygen in water negatively affected the accuracy of analyte determination. Potassium ferricyanide, with highly solubility in water $(46 \mathrm{~g} / 100 \mathrm{~mL})$, has been applied to an alternative chemical mediator to dissolved oxygen for electrochemical measurements. ${ }^{(11)}$ In the FC measurement, ascorbic acid (AsA; vitamin C) is well known as an electroactive interference, and this electrochemical property of AsA was applied to the antioxidant assay in the present study.

An increase in healthy life expectancy is one of the significant issues in the reduction in healthcare costs in Japan, and it is achieved by continuous control of physical conditions, e.g., eating habits. ${ }^{(12,13)}$ Antioxidants are involved in the defense mechanisms of an organism against the pathologies associated with the attack of free radicals. ${ }^{(14)}$ Thus, antioxidant properties of food have been a focus of attention recently. ${ }^{(15)}$ Many estimation methods for dietary antioxidants and total antioxidant capacity (TAC) have been developed, and most have employed optical methods.(16-18) An optical method using 2,2-diphenyl-1-picrylhydrazyl (DPPH) is commonly employed as a spectrophotometric method for the TAC assay. ${ }^{(19,20)}$ However, this method takes $20 \mathrm{~min}$ for color development. In addition, such an optical method requires expensive equipment and complex procedures, is time consuming, and is affected by light absorption and scattering substances in a food sample.

The use of biosensors is expected to be a simple and rapid method; however, an unstable biorecognition element is used and is only applicable to single-antioxidant measurements. ${ }^{(21-24)}$ As chemical sensor methods, electrochemical methods utilizing cyclic voltammetry (CV) and chemically modified electrode (CME) methods immobilizing a mediator have been actively studied in recent years. ${ }^{(2)}$ However, in general, the $\mathrm{CV}$ method was preferably utilized for qualitative measurements of various electrochemical characteristics. In addition, in both the CV and CME methods, an electrode system was basically repeatably used; therefore, the washing, regeneration 
treatment, and calibration of the device are required. In contrast, chronoamperometry (CA) is preferably utilized for quantitative measurement; therefore, it is better to accurately determine the TAC value using a disposable microchip.

It is known that vegetables contain antioxidants, such as AsA, chlorogenic acid (ChA), and $\alpha$-tocopherol ( $\alpha \mathrm{TP}$; vitamin E). AsA and ChA are hydrophilic, and $\alpha \mathrm{TP}$ is lipophilic. Both estimation and extraction methods depend on target antioxidants, and the antioxidant capacity values obtained from these methods have not been standardized. In particular, in the extraction method, both hydrophilic and lipophilic antioxidants have to be simultaneously extracted in many cases. Thus, as amphipathic solvents, methanol $(\mathrm{MeOH})$, ethanol $(\mathrm{EtOH})$, dimethyl sulfoxide (DMSO), $N, N$ dimethylformamide (DMF), $N, N$-dimethylacetamide (DMA), and others have been employed for antioxidant extraction from vegetables or other plant foods. ${ }^{(25-29)}$ In the present study, the solubility valance of both hydrophilic and lipophilic antioxidants was a key point for electrochemical antioxidant estimation using the ferricyanide mediator; therefore, extraction as well as FC measurement conditions were intensively investigated and optimized. Our aim in the present study is to standardize an estimation method for a vegetable TAC assay through a simple, rapid, and cost-effective FC measurement using a disposable microchip. To the best of our knowledge, this is the first report that describes an FC-TAC assay. In addition, our final goals are the standardization of the FC-TAC assay for foods and drinks and the popularization of the FC-TAC value for food labeling as well as calorie counts.

\section{Material and Methods}

\subsection{Materials}

Potassium hexacyanoferrate(II) (potassium ferricyanide), methanol (MeOH), L-ascorbic acid (AsA), chlorogenic acid (ChA), $\alpha$-tocopherol $(\alpha \mathrm{TP})$, and 1,1-diphenyl2-picrylhydrazyl (DPPH) were purchased from Wako Pure Chemicals (Osaka, Japan). The other chemicals used in this study were reagent grade. Trolox (6-hydroxy-2,5,7,8tetramethylchroman-2-carboxylic acid) was purchased from Merck Millipore Japan. Ultrapure water was used in the experiments. Potassium ferricyanide solution (290 mM) was prepared using the ultrapure water.

\subsection{FC measurement}

The FC measurements were performed using an electrochemical analyzer (Model: CHI-1202, BAS, Inc., US). The procedures for FC measurements are shown in Fig. 1. The microchip was connected to the electrochemical analyzer using a connector (see details in section 3.1). The sample solution $(27 \mu \mathrm{L})$ containing an antioxidant dissolved in $80 \%(\mathrm{v} / \mathrm{v}) \mathrm{MeOH}$ was dropped onto the electrode surface of the microchip, and the FC measurement was immediately started after dropping $3 \mu \mathrm{L}$ of $290 \mathrm{mM}$ potassium ferricyanide solution into the sample solution. A potential was applied to a working electrode (WE) at $0.8 \mathrm{~V}$ for $10 \mathrm{~s}$ after a $20 \mathrm{~s}$ rest. Finally, the current output obtained after $10 \mathrm{~s}$ application was used as the measured value. 


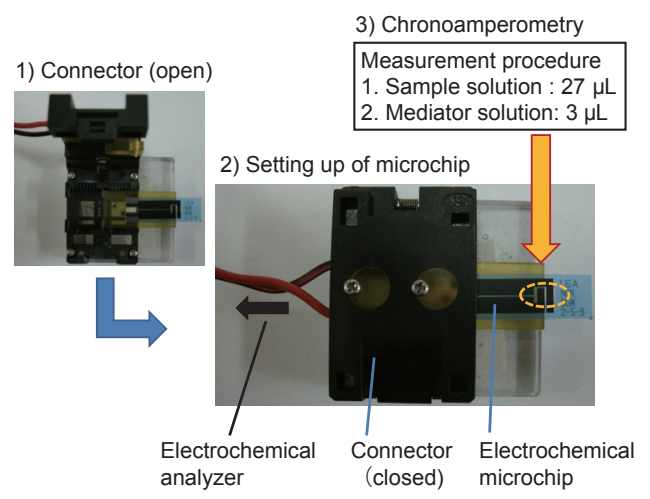

Fig. 1. (Color online) Procedures of the present FC measurement.

\subsection{Sample preparation and extraction of antioxidants}

For a real sample application, ten vegetables, namely cabbage, chive, crown daisy, cucumber, spinach, asparagus, lettuce, mizuna, onion, and radish sprouts, were chosen. Each vegetable was extracted under fresh or freeze-dried conditions using two solvents; i.e., four extraction samples for each vegetable were prepared and used in the experiments.

To obtain fresh samples, vegetables were washed with pure water, allowed to drain, and dried in a room for $1 \mathrm{~h}$. Subsequently, each vegetable was chopped into small pieces and weighed to $10 \mathrm{~g}$ using an electronic balance. After $5 \mathrm{~min}$ of vegetable milling using a muddler and a mortar, $20 \mathrm{~mL}$ of solvent was added to the milled vegetable. As solvents, $80 \%(\mathrm{v} / \mathrm{v})$ and $100 \%(\mathrm{v} / \mathrm{v}) \mathrm{MeOH}$ were used. The mixture was extracted for 1 $\mathrm{h}$ in an ultrasonic bath. The supernatant of the mixture was obtained after centrifugation at $13000 \mathrm{rpm}$ for $5 \mathrm{~min}$ and stored at $-30{ }^{\circ} \mathrm{C}$ under lightproof condition before use. Then, the supernatant sample extracted using $100 \%(\mathrm{v} / \mathrm{v}) \mathrm{MeOH}$ was diluted to $80 \%(\mathrm{v} / \mathrm{v})$ $\mathrm{MeOH}$ before storage.

To obtain freeze-dried samples, vegetables were washed, dried, chopped, and weighed to $10 \mathrm{~g}$ similarly to the case of fresh samples. The chopped vegetable was frozen at $-30{ }^{\circ} \mathrm{C}$ and freeze-dried at $-45^{\circ} \mathrm{C}$ and 20 Pa for $5 \mathrm{~d}$ using a lyophilizer (Model: FDU-1200, EYELA, Tokyo Rikakikai Co., Ltd., Japan). Both the freeze-dried samples were milled, extracted, and stored. Thus, the four samples from each vegetable extracted and the ten vegetables were used in both the present electrochemical method and the conventional DPPH test.

\subsection{DPPH test}

DPPH is a radical and a trap for radicals of antioxidants. The DPPH radical has a deep violet color in solution, and it becomes colorless when neutralized. This property allows visual monitoring of the reaction between DPPH and an antioxidant.

Trolox was used as the primary standard of antioxidant for the estimation of the trolox equivalent antioxidant capacity (TEAC) as in the conventional TAC assay. ${ }^{(19)}$ 
Trolox primary standard solutions were prepared by dissolving in $80 \%(\mathrm{v} / \mathrm{v}) \mathrm{MeOH}$ to $0,6.25,12.5,25,50$, and $100 \mu \mathrm{M}$ trolox, respectively. A solution $100 \mathrm{~mL}$ of $200 \mu \mathrm{M}$ $\mathrm{DPPH}$ dissolved in the MeOH solution was mixed with the same volume of the trolox primary standard solution in a 96-well microplate. The mixture was incubated for 20 min to develop color, and the absorbance of the mixture at $492 \mathrm{~nm}$ was measured using a microplate reader (Ultra Evolution, TECAN Group Ltd., Switzerland). On the basis of a calibration curve for trolox, TEAC values of AsA, ChA, and $\alpha \mathrm{TP}$ as secondary standards were calculated and used for the estimation of the antioxidant mixture and real samples, respectively.

\section{Results and Discussion}

\subsection{Principle of electrochemical ferricyanide method}

The electrochemical measurement for the TAC assay for vegetables was accomplished by a mediator-detecting system using potassium ferricyanide as an electron acceptor. The principle of the FC-TAC method, which is shown in Fig. 2, was to apply an oxidative potential to ferrocyanide, which is produced by reaction with antioxidants and ferricyanide on the electrode. The current was observed when ferrocyanide was electrochemically oxidized. The amount of ferrocyanide was proportional to the concentration of antioxidant.

\subsection{Microchip and connector}

For the evaluation of the TAC value by the FC measurement, a carbon screen-printed disposable microchip consisting of a two-electrode system was used. A polyethylene terephthalate (PET) sheet was used as a substrate of the electrode chip (length, $30 \mathrm{~mm}$; width, $10 \mathrm{~mm}$; thickness, $188 \mu \mathrm{m}$ ). Using a carbon screen printer, two electrodes were formed on the substrate at an interval of $0.5 \mathrm{~mm}$. Screen printing was also used to form a resist layer and define the area of each electrode as $3.75 \mathrm{~mm}^{2}$ for realizing an

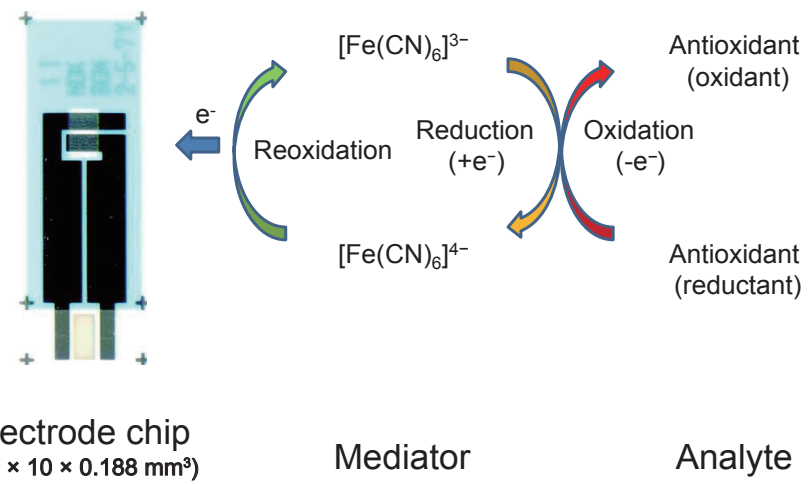

Fig. 2. (Color online) Principle of electrochemical measurement for antioxidant. 
electrochemical reaction. A connector between the microchip and the electrochemical analyzer was made-to-order as in a previous study. ${ }^{(6)}$ The microchips were used for onetime measurement only.

To investigate the electrochemical features of a carbon working electrode on a microchip, ferricyanide cyclic voltammetry (FCV) was utilized using a three-electrode system, which consists of the working, Pt counter, and $\mathrm{Ag} / \mathrm{AgCl}$ reference electrodes. These electrodes were dipped in a cuvette containing $10 \mathrm{mM}$ potassium ferrocyanide and $0.2 \mathrm{mM}$ sodium sulfate (total volume: $10 \mathrm{~mL}$ ). In the FCV measurements, the voltammogram was obtained at an initial potential at $0.2 \mathrm{~V}$, a high potential of $1.0 \mathrm{~V}$, a low potential of $0 \mathrm{~V}$, and a scan speed of $0.05 \mathrm{~V} / \mathrm{s}$. The features of the carbon electrode were compared with those of the Pt electrode (Fig. 3). The results indicate that the carbon electrode compared with the Pt electrode showed a highly oxidative peak at around $0.7 \mathrm{~V}$ to ferrocyanide with a wide potential window. It was also found that the carbon electrode had a lower reaction velocity than the Pt electrode. In addition, the roughness of the carbon electrode surface compared with that of the Pt electrode increased the surface area and induced an electrochemical response to a high current. The roughness of the carbon electrode surface was observed using a microscope (Fig. 4). Thus, the electrochemical features of the microchip were confirmed.

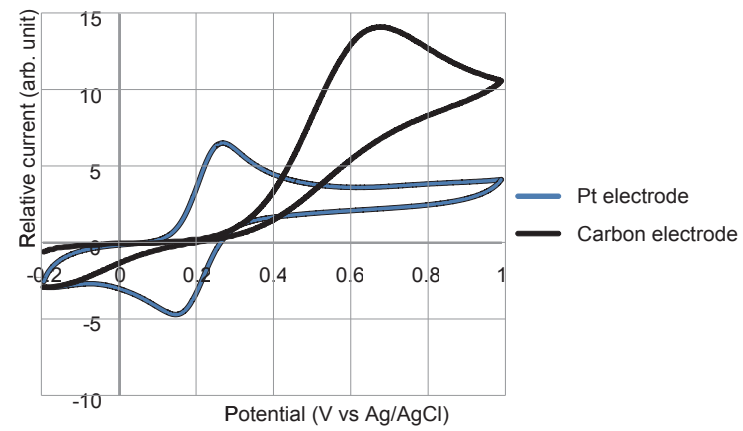

Fig. 3. (Color online) Comparison of the voltammogram between the carbon electrode and the $\mathrm{Pt}$ electrode.

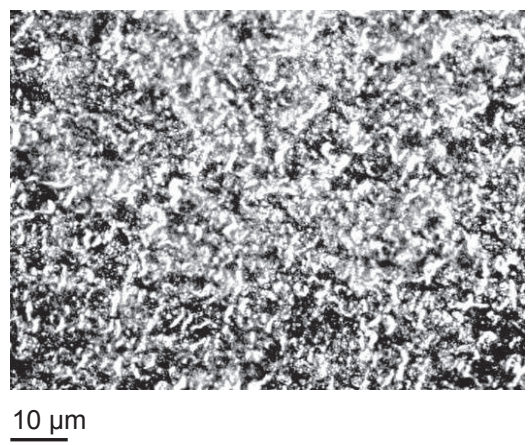

Fig. 4. Roughness of the carbon electrode surface. 


\subsection{Effects of ferricyanide mediator on antioxidant measurement}

Next, effects of the ferricyanide mediator on the antioxidant measurement using AsA were investigated by the FCV measurements. In the experiments, $5.8 \mathrm{~mL}$ of $1 \mathrm{mM}$ phosphate buffer solution ( $\mathrm{pH}$ 7.4) was mixed with $14.2 \mathrm{~mL}$ of each AsA solution. Their concentrations were as follows: $0,0.28,0.57,1.14,2.27$, and $4.57 \mathrm{mM}$ AsA. Then, the AsA solution was prepared using $60 \%(\mathrm{v} / \mathrm{v}) \mathrm{MeOH}$ solution as a solvent. The results were compared with those obtained using $10 \mathrm{mM}$ potassium ferricyanide dissolved in the same buffer. In the results obtained without the ferricyanide mediator, an oxidative peak of AsA was unclear owing to an overlap with that of water [Fig. 5(a)]. In contrast, an obvious oxidative peak of AsA was obtained using the ferricyanide mediator [Fig. 5(b)]. In addition, similar effects were also observed using ChA (data not shown). Thus, the effects of the ferricyanide mediator on the antioxidant measurement were clearly shown. Therefore, ferricyanide was used as the mediator in subsequent experiments.

\subsection{Optimization of FC measurement conditions for antioxidants}

\subsubsection{Potassium ferricyanide concentration}

For the optimization of the FC measurement conditions, the solution for potassium ferricyanide was first changed from phosphate buffer to pure water to increase the total solubility of the measurement solution. Next, the concentration of the ferricyanide mediator $(0,10,50,100,200$, and $400 \mathrm{mM} /$ water $)$ was investigated utilizing the FCV measurements and making calibration curves for $\operatorname{ChA}(0,0.25,0.5,1$, and $2 \mathrm{mM})$ with moderate solubility in three antioxidants. In the experiments, five repetitive measurements were performed. The results showed that the solubility of $400 \mathrm{mM}$ potassium ferricyanide in $60 \% \mathrm{MeOH}$ (about $40 \%$ at final concentration) was maintained; however, the standard deviation was markedly increased using potassium ferricyanide with a concentration above $200 \mathrm{mM}$, probably owing to the increase in initial current (data not shown). Therefore, $100 \mathrm{mM}$ potassium ferricyanide ( $29 \mathrm{mM}$ at final concentration) was chosen as a mediator, the sufficient electrochemical response of which to the antioxidant could be obtained in subsequent experiments.

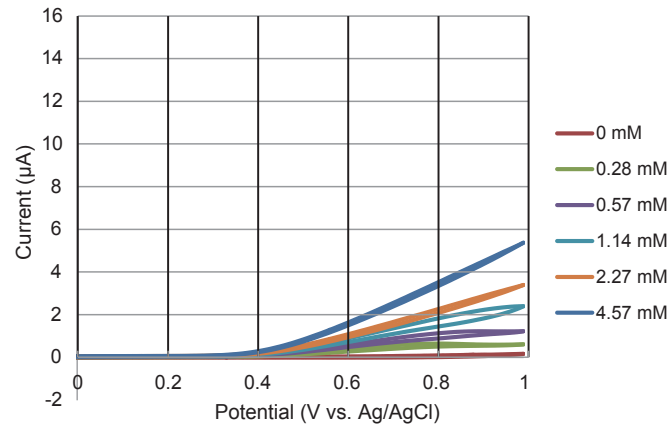

(a)

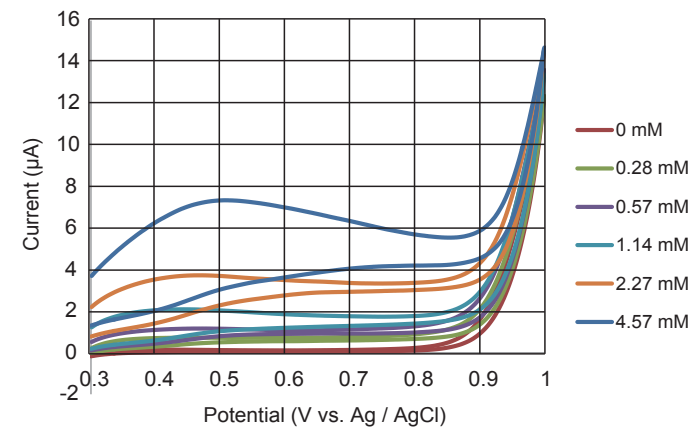

(b)

Fig. 5. (Color online) Effects of ferricyanide mediator on antioxidant response (AsA). Without and (b) with potassium ferricyanide. 


\subsubsection{Solubility valance estimation by visual evaluation}

The solubility valance of both hydrophilic and lipophilic antioxidants and potassium ferricyanide dissolved in the electrochemical measurement solution was subsequently investigated by visual evaluation in $10 \mathrm{~mL}$ of the stirring solution. In this investigation, $\mathrm{MeOH}, \mathrm{EtOH}, \mathrm{DMSO}, \mathrm{DMF}$, and DMA were used as amphiphatic solvents, and these solvents were used after dilution with pure water [50,60, 70, 80, 90, and $100 \%(\mathrm{v} / \mathrm{v})]$. The solubility of $0.5 \mathrm{mg} / \mathrm{mL}$ AsA, $0.5 \mathrm{mg} / \mathrm{mL} \mathrm{ChA,} 0.015 \mathrm{mg} / \mathrm{mL} \alpha \mathrm{TP}$, and 29 $\mathrm{mM}$ potassium ferricyanide as solutes dissolved in the electrochemical measurement solution was examined. Then the antioxidant concentrations examined were adopted by considering that hydrophilic conditions are desirable for electrochemical measurements using a hydrophilic mediator. However, $\alpha \mathrm{TP}$ is a lipophilic antioxidant. Therefore, the $\alpha \mathrm{TP}$ concentration examined was lower than (over 30 times) the water-soluble AsA and ChA concentrations. Nevertheless, the solubility of $\alpha \mathrm{TP}$ in the amphiphatic solvent decreased at a solvent concentration below $60 \%$. On the other hand, the solubility of potassium ferricyanide decreased at high concentrations of solvents, particularly EtOH (over $70 \%)$.

\subsubsection{Selection of solvent and optimization of electrochemical measurement conditions by FCV measurement}

Further investigations of the solubility valance of the antioxidants were carried out by FCV measurement using $80 \% \mathrm{MeOH}$, DMSO, DMF, and DMA without $\mathrm{EtOH}$. Then, the FCV measurement was carried out using a two-electrode system of the microchip. The antioxidant solution $(27 \mu \mathrm{L})$ dissolved in $80 \%$ solvent was dropped onto the surface of the microchip, and $3 \mu \mathrm{L}$ of $290 \mathrm{mM}$ potassium ferricyanide solution was subsequently dropped onto the antioxidant solution. The results obtained using AsA showed that the oxidative peak on the cyclic voltammogram was observed between 0.2 and $0.5 \mathrm{~V}$ for all solvents, and the clearest concentration dependence was observed below $1.42 \mathrm{mM}$ using $\mathrm{MeOH}$, although the concentration-dependent current was not obtained at $2.82 \mathrm{mM}$ AsA for the precipitation of the AsA.

In addition, the oxidative peak of ChA (to $2.82 \mathrm{mM}$ ) was observed at around $0.8 \mathrm{~V}$, and a ChA concentration dependence was observed at the oxidative peak using $\mathrm{MeOH}$, DMF, and DMA. However, an obvious peak due to ChA was not obtained using DMSO. In the response to $\alpha \mathrm{TP}$ (to $0.12 \mathrm{mM}$ ), a small current was observed at the oxidative peak between 0.3 and $0.6 \mathrm{~V}$, and a concentration-dependent current was also obtained at the oxidative peak in all solvents. From these results, it was found that an applied potential of more than $0.8 \mathrm{~V}$ for the FC measurement was suitable. However, such a high potential led to the oxidative decomposition of water. Thus, the optimum applied potential for the FC measurement was determined to be $0.8 \mathrm{~V}$.

\subsubsection{Confirmation of the optimum conditions for FC-TAC measurement}

The investigation of the solvent was continued by FC measurements. In the experiments, AsA, ChA, and $\alpha \mathrm{TP}$ were used as antioxidant standards. Their concentrations were as follows: $0,0.35,0.71,1.42$, and $2.84 \mathrm{mM}$ AsA; 0, 0.35, 0.71, 1.41 , and $2.82 \mathrm{mM} \mathrm{ChA}$; and $0,0.004,0.009,0.017$, and $0.035 \mathrm{mM} \alpha \mathrm{TP}$. A calibration curve for each standard was made using $\mathrm{MeOH}$, DMSO, DMF, and DMA solvents. 
Then $27 \mu \mathrm{L}$ of each antioxidant standard contained in $80 \%$ solvent and $3 \mu \mathrm{L}$ of $290 \mathrm{mM}$ potassium ferricyanide solution were mixed on the microchip. In the FC measurement, a potential of $0.8 \mathrm{~V}$ was applied to the WE for $10 \mathrm{~s}$ after a $20 \mathrm{~s}$ rest. After $10 \mathrm{~s}$ application, the current output obtained was used as the measured FC value. A representative response curve of the FC measurements is shown in Fig. 6.

All of the results presented in this section are summarized in Table 1. In the FC measurement, a large slope in a linear regression curve was obtained for all antioxidants dissolved in $\mathrm{MeOH}$ ( $80 \%$; final concentration $72 \%$ ), probably owing to the fact that $\mathrm{MeOH}$ is known as a protonic solvent and is effective in stabilizing dissolved ions. ${ }^{(25)}$ By the encompassing investigations, the preferred solubility of both antioxidants and potassium ferricyanide was finally determined, and an $80 \%(\mathrm{v} / \mathrm{v}) \mathrm{MeOH}$ solution was used as a solvent for the FC-TAC assay.

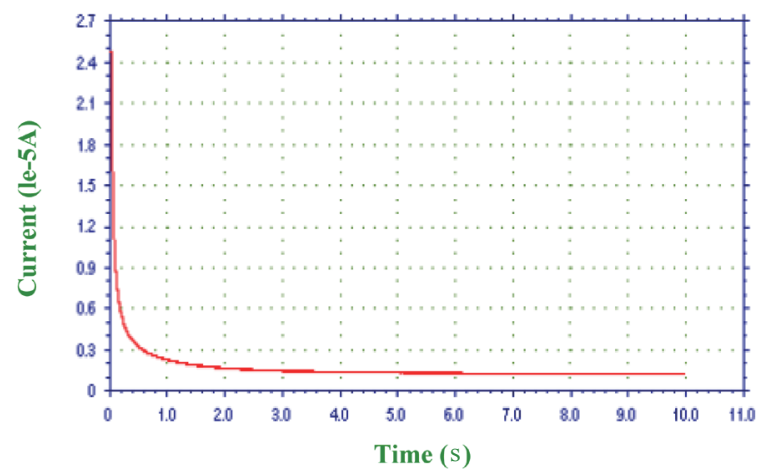

Fig. 6. (Color online) Representative response curve of the FC measurements.

Table 1

Comparison of the solvents used in the FC method $(n=5)$.

\begin{tabular}{|c|c|c|c|c|}
\hline \multirow{3}{*}{$\begin{array}{l}\text { Standard } \\
\text { solvent }[80 \%(\mathrm{v} / \mathrm{v})] \\
\text { AsA }\end{array}$} & \multirow{2}{*}{$\begin{array}{c}\text { Number of plots } \\
(n)\end{array}$} & \multicolumn{2}{|c|}{ Linear regression curve } & \multirow{2}{*}{$\begin{array}{l}\text { Determination coefficient } \\
\qquad\left(r^{2}\right)\end{array}$} \\
\hline & & Slope $(\mu \mathrm{A} / \mathrm{mM})$ & $y$-intercept $(\mu \mathrm{A})$ & \\
\hline & & & & \\
\hline $\mathrm{MeOH}$ & 5 & 3.7 & 0.65 & 0.986 \\
\hline DMSO & 5 & 2.3 & 0.26 & 0.939 \\
\hline DMF & 5 & 0.57 & 0.48 & 0.994 \\
\hline DMA & 5 & 0.91 & 0.40 & 0.979 \\
\hline \multicolumn{5}{|l|}{ ChA } \\
\hline $\mathrm{MeOH}$ & 5 & 3.3 & 0.51 & 0.996 \\
\hline DMSO & 5 & 0.35 & 0.12 & 0.983 \\
\hline DMF & 5 & 1.6 & 0.57 & 0.989 \\
\hline DMA & 5 & 1.9 & 0.47 & 0.981 \\
\hline \multicolumn{5}{|l|}{$\alpha \mathrm{TP}$} \\
\hline $\mathrm{MeOH}$ & 5 & 4.6 & 0.29 & 0.989 \\
\hline DMSO & 5 & 2.6 & 0.10 & 0.910 \\
\hline DMF & 5 & 5.3 & 0.48 & 0.989 \\
\hline DMA & 5 & 2.0 & 0.40 & 0.654 \\
\hline
\end{tabular}




\subsection{Comparison between present FC-TAC method and conventional DPPH test}

\subsubsection{Standard solution}

Under optimum conditions, the response to the antioxidant in the FC measurement was investigated using three standard antioxidant solutions, i.e., AsA, ChA, and $\alpha \mathrm{TP}$, at six different concentrations. The standard solutions of AsA and ChA were prepared to be $0,6.25,12.5,25,50$, and $100 \mathrm{mM}$ using $80 \% \mathrm{MeOH}$ solution, and the standard solutions of $\alpha \mathrm{TP}$ were prepared to be $0,0.004,0.009,0.017,0.035$, and $0.070 \mathrm{mM}$ in the same manner. Standard antioxidant mixtures containing AsA, ChA, and $\alpha \mathrm{TP}$ imitating vegetable components were also prepared. The components of three antioxidants in vegetables are shown in Table 2. . $^{(30,31)}$

In the DPPH radical scavenging capacity test, TEAC values of AsA, ChA, and $\alpha \mathrm{TP}$ as secondary standards were investigated using their standard solutions. AsA and ChA were diluted 40 times for the FC measurement, i.e., $0,0.156,0.313,0.625,1.25$, and $2.5 \mathrm{mM}$, using $80 \% \mathrm{MeOH}$. The $\alpha \mathrm{TP}$ standard and standard mixture were used without dilution.

The results obtained with both methods were compared with a linear regression curve between FC values $(\mu \mathrm{A})$ and trolox equivalent $(\mu \mathrm{M})$, which are shown in Table 3. The slopes of the linear regression curves were close to $2.8 \mu \mathrm{A} / \mathrm{mM}$, and excellent determination coefficient $\left(r^{2}\right)$ values of over 0.99 were observed for three pure antioxidants. Thus, the response to each antioxidant obtained by the FC measurement was obtained equivalently from three antioxidants without any effects of solubility on the solvent. In addition, a good $r^{2}$ value of 0.98 was observed using the standard antioxidant mixture imitating vegetable components (Fig. 7). Thus, favorable results between both methods were obtained using standard antioxidant solutions. These results obtained by

Table 2

Constituents of three antioxidants in vegetables.

\begin{tabular}{lccc}
\hline & \multicolumn{3}{c}{ Antioxidants $(\mathrm{mg} / 100 \mathrm{~g})$} \\
\cline { 2 - 4 } & $\mathrm{AsA}^{(30)}$ & $\mathrm{ChA}^{(31)}$ & $\alpha \mathrm{TP}^{(30)}$ \\
\hline a cabbage & 41 & 10 & 0.1 \\
b spinach & 35 & 1 & 2.1 \\
c crown daisy & 19 & 33 & 1.7 \\
d chive & 19 & 2 & 2.5 \\
e cucumber & 14 & 4 & 0.3 \\
\hline
\end{tabular}

No dilution: a, b, c, d, and e; two-fold dilution: a', b', c', d', and e'; four-fold dilution: a", b", c", d", and e" (see Fig. 7).

Table 3

Comparison between the present FC method and the conventional DPPH test $(n=5)$.

\begin{tabular}{lcccc}
\hline Standard & $\begin{array}{c}\text { Number of plots } \\
(n)\end{array}$ & $\begin{array}{c}\text { Linear regression curve } \\
\text { Slope }(\mu \mathrm{A} / \mathrm{mM})\end{array}$ & $y$-intercept $(\mu \mathrm{A})$ & $\begin{array}{c}\text { Determination coefficient } \\
\left(r^{2}\right)\end{array}$ \\
\hline antioxidant(s) & & & & \\
AsA & 6 & 2.7 & 0.39 & 0.994 \\
ChA & 6 & 2.8 & 0.17 & 0.993 \\
$\alpha \mathrm{TP}$ & 6 & 2.6 & 0.30 & 0.999 \\
mixture* & 15 & 3.0 & 0.20 & 0.980 \\
\hline
\end{tabular}

*See Table 2 . 


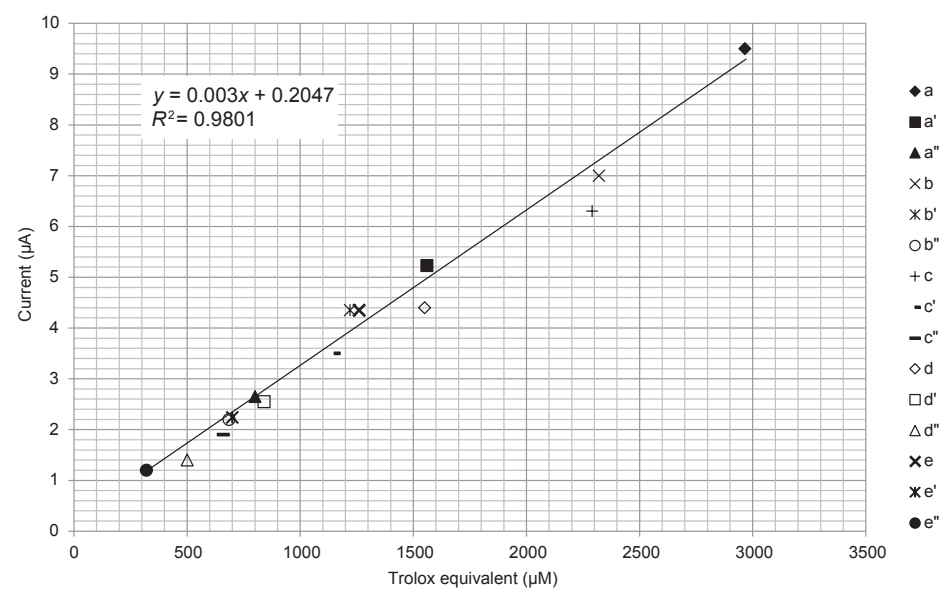

Fig. 7. Response to antioxidant mixture close to vegetable components. In Table 2, explanations of legends are shown. ${ }^{(30,31)}$

the present FC measurements sufficiently showed the possibility of applying the TAC assay to real sample estimation as an alternative to the conventional DPPH test.

\subsubsection{Real sample}

Real sample applications using vegetable extractions were performed using the FC measurements, and the results of the TAC assay were compared with those obtained by the DPPH test employing the trolox equivalent method (Fig. 8). In the experiments, ten vegetables, i.e., radish sprouts (Raphanus sativus var. longipinnatus), cabbage (Brassica oleracea var. capitata), lettuce (Lactuca sativa), asparagus (Asparagus spp.), mizuna (Brassica rapa var. nipposinica), spinach (Spinacia oleracea), onion (Allium cepa), cucumber (Cucumis sativus), chive (Allium tuberosum), and crown daisy (Glebionis coronaria), were extracted using $\mathrm{MeOH}$, which was found to be the best solvent for the FC measurement (see $\S 3.4$ ). Figures $8(\mathrm{a})$ and $8(\mathrm{~b})$ show very similar results obtained by the $80 \% \mathrm{MeOH}$ or $100 \% \mathrm{MeOH}$ extraction of fresh vegetables. On the other hand, the results obtained using freeze-dried samples showed relatively higher responses than those obtained using fresh samples [Figs. 8(c) and 8(d)], and the highest responses were observed for $80 \% \mathrm{MeOH}$ extraction. The response value differences between these extraction conditions were observed, probably owing to the intrinsic water content in the fresh vegetables and high amounts of electrolytes extracted from the freeze-dried vegetables using $80 \% \mathrm{MeOH}$.

Compared with the results obtained using standard solutions, an approximately 2.3 -fold reduction in the slope of the linear regression curves was observed from that obtained using real sample solutions. Two major reasons for this were considered. One is that the reactive properties of antioxidants in real samples might be different between two measurement methods, and another is that other components in real samples effect the two measurement methods differently. 


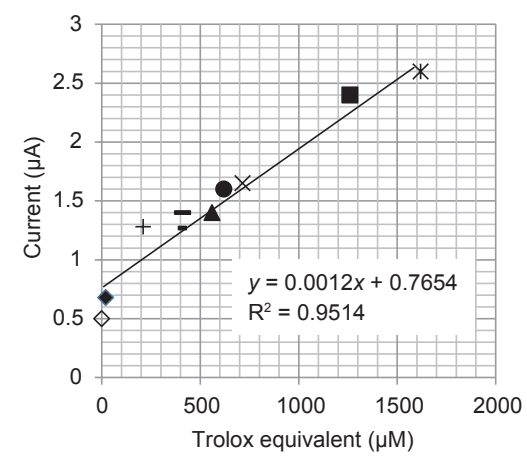

(a)

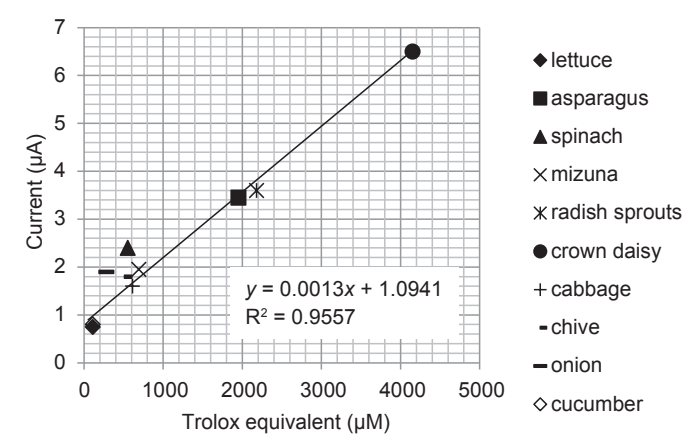

(c)

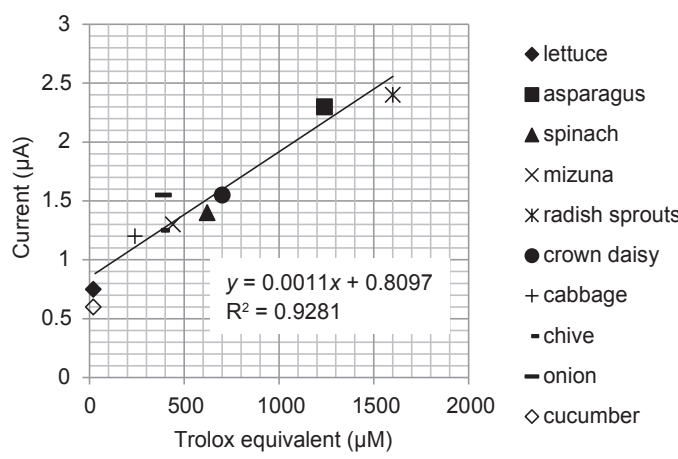

(b)

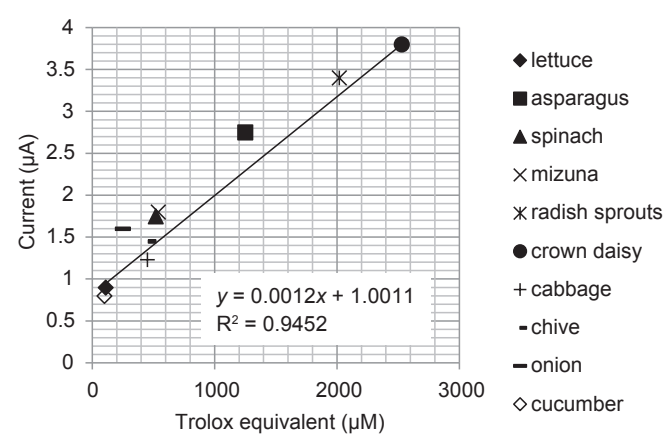

(d)

Fig. 8. Comparison of the TAC assays between the present FC method and the conventional DPPH test by the trolox equivalent method. (a) $80 \% \mathrm{MeOH}$ extraction of fresh vegetables, (b) $100 \% \mathrm{MeOH}$ extraction of fresh vegetables, (c) $80 \% \mathrm{MeOH}$ extraction of freeze-dried vegetables, and (d) $100 \% \mathrm{MeOH}$ extraction of freeze-dried vegetables.

In addition, a relatively high and unexpected response during the FC measurements was observed using onion in all pretreatments [Figs. 8(a)-8(d)] and spinach in freezedried pretreatments [Figs. 8(c) and 8(d)]. In contrast, relatively low responses, which are probably due to the ascorbic oxidase activity, were observed using lettuce and cucumber in all pretreatments, [Figs. 8(a)-8(d)].

On the other hand, no obvious determination coefficient differences were observed in the pretreatments using extracting conditions (fresh or freeze-dried condition) or extracting solvents [Figs. 8(a)-8(d)]. Thus, the possibility of the present electrochemical method as the simple, rapid, and cost-effective estimation method was successfully shown by these results. 


\section{Conclusions}

In the present study, we have developed a screen-printed disposable microchip and established a standardized FC-TAC assay method for vegetables employing a $\mathrm{MeOH}$ solvent for both FC measurements and vegetable extractions. A small sample volume, a simple measurement procedure, and a rapid and accurate FC measurement using a microstructured disposable chip were other beneficial characteristics of the present TAC assay, although minor effects of undefined substances in both onion and spinach and electrolytes in vegetables were observed. To examine the effects on the results, further examination is now being planned. Thus, the possibility of expanding the present FCTAC assay to expand to other kinds of foods and drinks is considered. The next step in expanding the study is in progress for practical use.

\section{References}

1 H. Nakamura and I. Karube: Anal. Bioanal. Chem. 377 (2003) 446.

2 H. Nakamura, M. Suda, Y. Murakami, K. Yokoyama, E. Tamiya, S. Uchiyama and I. Karube: Anal. Chem. 73 (2001) 373.

3 F. Kurusu, T. Ishikawa, S. Koide, S. Kaimori, M. Ichino, T. Hosoya, H. Nakamura, M. Gotoh and I. Karube: JJCLA (issued by "The Japan Society for Clinical Laboratory Automation") 30 (2005) 210.

4 T. Kitamura, S. Kaimori, A. Harada, T. Ishikawa, T. Fujimura, H. Nakamura, M. Gotoh and I. Karube: SEI Tech. Rev. 63 (2006) 19.

5 S. Kaimori, T. Kitamura, M. Ichino, T. Hosoya, F. Kurusu, T. Ishikawa, H. Nakamura, M. Gotoh and I. Karube: Anal. Chim. Acta 573-574 (2006) 104.

6 H. Nakamura, K. Tohyama, M. Tanaka, S. Shinohara, Y. Tokunaga, F. Kurusu, S. Koide, M. Gotoh and I. Karube: Biosens. Bioelectr. 23 (2007) 621.

7 H. Nakamura, R. Tanaka, K. Suzuki, M. Yataka and Y. Mogi: Food Chem. 117 (2009) 509.

8 H. Nakamura, K. Suzuki, H. Ishikuro, S. Kinoshita, R. Koizumi, S. Okuma, M. Gotoh and I. Karube: Talanta 72 (2007) 210.

9 N. Yoshida, J. Hoashi, T. Morita, S. J. McNiven, K. Yano, A. Yoshida, H. Nakamura and I. Karube: Analyst 126 (2001) 1751.

10 S. J. Updike and G. P. Hicks: Nature 214 (1967) 986.

11 G. Bardeletti, F. Sechaud and P. R. Coulet: Biotechnology and Bioprocessing, eds. L. J. Blum and P. R. Coulet (Marcel Dekker Inc., New York, 1991) Vol. 15, Chap. 2.

12 Current Status and Problem of Health Insurance in Our Country: White Paper (Ministry of Health, Labour and Welfare, Japan, 2007) Chap. 2.

13 Aged Society: White Paper (Cabinet Office, Japan, 2008).

14 T. P. A. Devasagayam, J. C. Tilak, K. K. Boloor, K. S. Sane, S. S. Ghaskadbi and R. D. Lele: JAPI 52 (2004) 794.

15 D. B. Haytowitz and S. Bhagwat: USDA Database for the Oxygen Radical Absorbance Capacity (ORAC) of Selected Foods (USDA, Washington, D.C., 2010) Release 2.

16 W. Brand-Williams, M. E. Cuvelier and C. Berset: Food Sci. Tech. 28 (1995) 25.

17 G. Bartosz: Total Antioxidant Capacity, ed. H. Spiegel (Advances in Clinical Chemistry, Vol. 37), (Academic Press, USA, 2003) p. 219.

18 A. M. Pisoschi and G. P. Negulescu: Biochem. Anal. Biochem. 1 (2011) 106.

19 O. P. Sharma and T. K. Bhat: Food Chem. 113 (2009) 1202. 
20 C. López-Alarcóna and A Denicolab: Anal. Chim. Acta 763 (2013) 1.

21 L. D. Mello and L. T. Kubota: Talanta 72 (2007) 335.

22 M. F. Barrosoa, N. de-los-Santos-Álvarezc, C. Delerue-Matosa and M. B. P. P. Oliveira: Biosens. Bioelectr. 30 (2011) 1.

23 A. Barberis, Y. Spissu, G. Bazzu, A. Fadda, E. Azara, D. Sanna, M. Schirra and P. A. Serra: Anal. Chem. 86 (2014) 8727.

24 A. Barberis, Y. Spissub, A. Faddaa, E. Azarac, G. Bazzub, S. Marceddua, A. Angionid, D. Sannac, M. Schirraa and P. A. Serrab: Biosens. Bioelectr. (in press).

25 C. K. Ramesh, K. L. Raghu, K. S. Jamuna, G. S. Joyce, R-S. Vijayanti Mala and B. R. V. Avin: Ann. Biol. Res. 2 (2011) 86.

26 M. R. S. Ardekani, M. Khanavi, M. Hajimahmoodi, M. Jahangiria and A. Hadjiakhoodi: J. Pharmaceut. Res. 9 (2010) 141.

27 L. Wu: J. Zhejiang Univ. Sci. B 8 (2007) 673.

28 N. Turkmen, F. Sari and Y. Sedat Velioglu: Food Chem. 99 (2006) 835.

29 A. Ismail and S. F Cheah: Malays. J. Nutr. 9 (2003) 31.

30 Food composition database (Ministry of Education, Culture, Sports, Science and Technology, Japan, 2012).

31 Nutrient composition and polyphenol content of sugar cane leaf for foliage usage (Kyushu Agricultural Research Center, Japan, 1999). 